\title{
ASSOCIATE INVERSE SUBSEMIGROUPS OF REGULAR SEMIGROUPS
}

\author{
B. BILLHARDT, E. GIRALDES, P. MARQUES-SMITH, P. MENDES MARTINS
}

\begin{abstract}
By an associate inverse subsemigroup of a regular semigroup $S$ we mean a subsemigroup $T$ of $S$ containing a least associate of each $x \in S$, in relation to the natural partial order $\leq_{S}$. We describe the structure of a regular semigroup with an associate inverse subsemigroup, satisfying two natural conditions. As a particular application, we obtain the structure of regular semigroups with an associate subgroup with medial identity element.
\end{abstract}

\section{INTRODUCTION}

In [3] the authors introduced the notion of an associate subgroup of an orthodox semigroup $S$. This is a subsemigroup $T$ of $S$ with the property that $|T \cap A(x)|=1$, for all $x \in S$, where $A(x)=\{y \in S: x=x y x\}$ is the set of associates of $x$. Such a semigroup is necessarily a group, specifically, the maximal subgroup $H_{\alpha}$ of $S$, where $\alpha$ is the identity element of the group [[3], Theorem 3]. The main result of [3] is a complete description of the structure of an orthodox semigroup $S$ with an associate subgroup whose identity element $\alpha$ is a middle unit (i.e., $x \alpha y=x y$, for every $x, y \in S$ ). Taking into account that in an orthodox semigroup with an associate subgroup the identity $\alpha$ of the group is medial (i.e., $x \alpha x=x$, for all $x \in\langle E(S)\rangle)$ [[3], Theorem 5], Blyth and Mendes Martins described in [4] the structure of regular semigroups with an associate subgroup whose identity element is a medial idempotent, thus obtaining a generalization of the work developed in [3]. We begin this paper by introducing the concept of an associate inverse subsemigroup, extending the notion of associate subgroup of a semigroup. An associate inverse subsemigroup of a regular semigroup is a subsemigroup $T$ of $S$ containing a least associate of each $x \in S$, in relation to the natural partial order $\leq_{S}$ in $S$. Such a subsemigroup $T$ of $S$ is necessarily inverse. The motivation of this study lies in a very simple and interesting characterization of inverse semigroups in terms of the natural partial order in an arbitrary semigroup. Semigroups with inverse associate subsemigroups occur naturally in the context of inverse transversals. These are inverse subsemigroups of a semigroup $S$ that contain exactly a single inverse of each $s \in S$. Inverse transversals have been extensively studied by many mathematicians. A review of the theory can be found in [2]. While in some cases inverse

2000 Mathematics Subject Classification. Primary 20M10, 20M17.

Key words and phrases. Regular semigroup, associate inverse subsemigroup, inverse transversal.

Research supported by the Portuguese Foundation for Science and Technology (FCT) through the research program POCTI. 
transversals and inverse associate subsemigroups coincide, in many important classes of semigroups these two concepts are not even related (see section 3 of this paper). This reality provides further motivation for this study.

In section 2 we present an axiomatic characterization of semigroups with an associate inverse subsemigroup and in section 3 the connection with inverse transversals is explored. Finally, in section 4, we establish the structure of regular semigroups with an associate inverse subsemigroup, satisfying two natural conditions, and deduce an application of the theorem to obtain the structure of regular semigroups that have an associate subgroup with medial identity element.

For standard notions and notation in semigroup theory we refer the reader to the textbooks of Howie [5] Lawson [6] and Petrich [8]. In particular, for a semigroup $S, E(S)$ denotes the subset of idempotents of $S$ and $\langle E(S)\rangle$ represents the subsemigroup generated by $E(S)$. Moreover, if $S$ is inverse, the natural partial order $\leq$ in $S$ is defined by

$$
x \leq y \Longleftrightarrow x=e y,
$$

for some $e \in E(S)$. If $S$ is regular $\leq$ is extended by

$$
x \leq y \Longleftrightarrow x=y e=f y,
$$

for some $e, f \in E(S)$. Throughout the paper, all order related concepts and comments are considered with respect to the natural partial order.

\section{Associate inverse subsemigroups}

We begin with a characterization of inverse semigroups in terms of the natural partial order in a semigroup. This proved to be of fundamental importance for the subsequent results.

Theorem 2.1. Let $S$ be a regular semigroup. The following are equivalent:

(i) $S$ is inverse;

(ii) For all $a \in S, A(a)$ contains a least element with respect to the natural partial order.

Proof. $(i) \Rightarrow(i i)$. Let $a \in S$. Since $a a^{-1} a=a, a^{-1} \in A(a)$ and so $A(a) \neq \emptyset$. We show that $a^{-1}$ is the least element of $A(a)$. Let $x \in A(a)$. Since $a=a x a$ and $S$ is inverse, $a^{-1}=(a x a)^{-1}=a^{-1}(a x)^{-1}=a^{-1} a x$. Thus $a^{-1} \leq x$.

$(i i) \Rightarrow(i)$. Since the semigroup $S$ is regular, the natural partial order in $S$ is given by

$$
a \leq b \Leftrightarrow a=f b=b e, \text { for some idempotents } e, f \in E(S) .
$$

Let $a \in S$ and let

$$
a^{*}=\min \{x \in S: a x a=a\}
$$


We show that $a^{*}$ is the unique inverse of $a$ in $S$. On one hand, since $a^{*} a a^{*} \in V(a) \subseteq A(a)$, $a^{*} \leq a^{*} a a^{*}$. On the other hand, since both $a^{*} a$ and $a a^{*}$ are idempotents, we have that $a^{*} a a^{*} \leq a^{*}$. Thus $a^{*} a a^{*}=a^{*} \in V(a)$. Next let $a^{\prime}$ be an inverse of $a$. By hypothesis, there exist idempotents $e, f \in S$ such that $a^{*}=a^{\prime} e=f a^{\prime}=a^{*} e=f a^{*}$ and so

$$
a^{\prime} a a^{*}=a^{\prime} a a^{\prime} e=a^{\prime} e=a^{*}
$$

and

$$
a^{*} a a^{\prime}=f a^{\prime} a a^{\prime}=f a^{\prime}=a^{*} .
$$

Hence,

$$
a^{*}=a^{\prime} a a^{*}=a^{\prime} a a^{*} a a^{\prime}=a^{\prime} a a^{\prime}=a^{\prime} .
$$

Thus $a^{*}$ is the unique inverse of $a$ in $S$.

Let $S$ be a regular semigroup and $T$ a subsemigroup of $S$ such that, for each $s \in S$, $A(s) \cap T$ has a least element with respect to the natural partial order. By Theorem 2.1, the subsemigroup $T$ of $S$ is inverse. We call such a semigroup $T$ an associate inverse subsemigroup of $S$ and denote it by $S^{*}$. For each $s \in S$, we denote the least element of $A(s) \cap T$ by $s^{*}$. We also define $x^{* *}=\left(x^{*}\right)^{*}$ for every $x \in S$. Then $\left(x^{*}\right)^{* *}=\left(\left(x^{*}\right)^{*}\right)^{*}=\left(x^{* *}\right)^{*}$ and so we can write as $x^{* * *}$.

Proposition 2.2. Let $S$ be a regular semigroup with an associate inverse subsemigroup $S^{*}$. For each $s, t \in S$, the following hold

(i) $s^{*} s^{* *} t^{*} t^{* *}=t^{*} t^{* *} s^{*} s^{* *}$;

(ii) $s^{* * *}=s^{*}$;

(iii) $(s t)^{*} \leq t^{*}\left(s^{*} s t t^{*}\right)^{*} s^{*}$.

Proof. (i) For $s, t \in S, s^{*} s^{* *}$ and $t^{*} t^{* *}$ are idempotents of the associate inverse subsemigroup. Since this semigroup is inverse, they commute.

(ii) Since the associate subsemigroup is inverse, for $s \in S$, it follows from the proof of Theorem 2.1 that $s^{* *}=\left(s^{*}\right)^{-1}$ and so

$$
s^{* * *}=\left[\left(s^{*}\right)^{-1}\right]^{-1}=s^{*} .
$$

(iii) For $s, t \in S$,

$$
s t \cdot t^{*}\left(s^{*} s t t^{*}\right)^{*} s^{*} \cdot s t=s s^{*} s t t^{*}\left(s^{*} s t t^{*}\right)^{*} s^{*} s t t^{*} t=s s^{*} s t t^{*} t=s t .
$$

Then, $t^{*}\left(s^{*} s t t^{*}\right)^{*} s^{*} \in S^{*} \cap A(s t)$ and so, by definition of $(s t)^{*}$, we obtain

$$
(s t)^{*} \leq t^{*}\left(s^{*} s t t^{*}\right)^{*} s^{*} .
$$

Proposition 2.3. Let $S$ be a regular semigroup with an associate inverse subsemigroup $S^{*}$. The assignment $x \mapsto x^{*}$ is a unary operation on $S$ that satisfies, for all $s, t \in S$,

(i) $s=s s^{*} s$;

(ii) $s^{*} t^{*}=\left(s^{*} t^{*}\right)^{* *}$; 
(iii) $s=s t^{*} s \Rightarrow s^{*} \leq t^{*}$.

Proof. (i) Clear from the definition of $s^{*} \in S^{*} \cap A(s)$.

(ii) Let $s^{*}, t^{*} \in S^{*}$. Since the semigroup $S^{*}$ is inverse, $s^{*} t^{*}=\left[\left(s^{*} t^{*}\right)^{-1}\right]^{-1}$. Theorem 2.1 now gives $s^{*} t^{*}=\left[\left(s^{*} t^{*}\right)^{*}\right]^{*}$.

(iii) Clear from the definition of $s^{*}$.

Proposition 2.4. Let $S$ be a regular semigroup with a unary operation $x \mapsto x^{\prime}$ satisfying (i), (ii) and (iii) of Proposition 2.3. Then $S^{\prime}=\left\{x^{\prime}: x \in S\right\}$ is an associate inverse subsemigroup of $S$.

Proof. Let $x \in S$. Then, by (i), $x^{\prime} \in A(x)$ and so $x^{\prime} \in A(x) \cap S^{\prime}$. Moreover, if $t^{\prime} \in A(s) \cap S^{\prime}$ then $x=x t^{\prime} x$ and (iii) implies that $x^{\prime} \leq t^{\prime}$. So $x^{\prime}$ is the least associate of $x$ in $S^{\prime}$. By (ii) we have that $S^{\prime}$ is a subsemigroup of $S$. It now follows from Theorem 2.1 that the semigroup $S^{\prime}$ is inverse. Thus, it is an associate inverse subsemigroup of $S$.

An axiomatic characterization of a semigroup containing an associate inverse subsemigroup is a consequence of Propositions 2.3 and 2.4.

Theorem 2.5. A regular semigroup $S$ contains an associate inverse subsemigroup if and only if it has a unary operation $x \mapsto x^{*}$ satisfying, for all $s, t \in S$,

(i) $s=s s^{*} s$;

(ii) $s^{*} t^{*}=\left(s^{*} t^{*}\right)^{* *}$;

(iii) $s=s t^{*} s \Rightarrow s^{*} \leq t^{*}$.

\section{The INVERSE TRANSVERSAL CONNECTION}

An inverse transversal of a regular semigroup $S$ is an inverse subsemigroup $S^{\circ}$ of $S$ such that $\left|S^{\circ} \cap V(s)\right|=1$, for all $s \in S$. We denote the only element of $S^{\circ} \cap V(s)$ by $s^{\circ}$.

In any regular semigroup with inverse transversal $S^{\circ}$, for all $a \in S, a^{\circ}$ is a minimal associate of $a$ in $S^{\circ}$. In fact, if $t^{*} \in A(a) \cap S^{\circ}$ and $t^{*} \leq a^{\circ}$ then $a^{\circ} e=t^{*}=f a^{\circ}$, for some $e, f \in E(S)$. Thus, $t^{*} a t^{*}=f a^{\circ} a a^{\circ} e=f a^{\circ} e=t^{*}$, whence $t^{*} \in V(a) \cap S^{\circ}$ and so $t^{*}=a^{\circ}$.

In general, for a regular semigroup $S$ having both an inverse transversal and an associate inverse subsemigroup, these two inverse subsemigroups of $S$ are not related. In some classes of semigroups, special kinds of inverse transversals are, however, intimately related to associate inverse subsemigroups. Weakly multiplicative inverse transversals are an example.

An inverse transversal $S^{\circ}$ of a regular semigroup $S$ is said to be

- weakly multiplicative if $\left(e \in E(S) \Rightarrow e^{\circ} \in E\left(S^{\circ}\right)\right)$; 
- associate if it is an associate inverse subsemigroup of $S$.

Proposition 3.1. Any weakly multiplicative inverse transversal of a regular semigroup $S$ is associate.

Proof. Let $S$ be a regular semigroup with weakly multiplicative inverse transversal $S^{\circ}$. Let $a \in S$ and $b^{\circ} \in A(a) \cap S^{\circ}$. Then $a=a b^{\circ} a$. We have, by [[2], Theorems 1.1 and 1.4],

$$
\begin{aligned}
a=a b^{\circ} a & \Rightarrow a^{\circ}=\left(\left(a b^{\circ}\right)^{\circ} a b^{\circ} a\right)^{\circ}\left(a b^{\circ}\right)^{\circ} \Rightarrow a^{\circ}=\left(\left(a b^{\circ}\right)^{\circ} a\right)^{\circ} b^{\circ \circ} a^{\circ} \\
& \Rightarrow a^{\circ}=\left(b^{\circ \circ} a^{\circ} a\right)^{\circ} b^{\circ \circ} a^{\circ} \Rightarrow a^{\circ}=a^{\circ} a^{\circ \circ} b^{\circ} b^{\circ \circ} a^{\circ} \\
& \Rightarrow a^{\circ}=b^{\circ} b^{\circ \circ} a^{\circ} a^{\circ \circ} a^{\circ} \Rightarrow a^{\circ}=b^{\circ} b^{\circ \circ} a^{\circ} \\
& \Rightarrow a^{\circ}=b^{\circ}\left(a b^{\circ}\right)^{\circ} \Rightarrow a^{\circ} \in b^{\circ} E\left(S^{\circ}\right) \Rightarrow a^{\circ} \leq b^{\circ} .
\end{aligned}
$$

Corollary 3.2. Let $S$ be a regular monoid. An inverse transversal $S^{\circ}$ of $S$ is associate if and only if it is weakly multiplicative.

Proof. Suppose that $S^{\circ}$ is associate and let $e \in E(S)$. Then, from the observation at the beginning of this section, $e^{*}=e^{\circ}$. Since $S$ has identity $1_{S}$ and $1_{S} \in A(e) \cap S^{*}$, it follows that $e^{*} \leq 1_{S}$, i.e., $e^{\circ} \leq 1_{S}$ and so $e^{\circ} \in E\left(S^{\circ}\right)$. Thus $S^{\circ}$ is weakly multiplicative.

The converse follows from the previous proposition.

Proposition 3.3. Let $S$ be a regular semigroup and $T$ a subsemigroup of $S$ satisfying

$$
t S t \subseteq T, \text { for all } t \in T .
$$

Then $T$ is an inverse transversal if and only if $T$ is an associate inverse subsemigroup of $S$.

Proof. Suppose that $T$ is an inverse transversal of a regular semigroup $S$ such that $t S t \subseteq T$, for all $t \in T$. Let $s \in S$ and $s^{\circ}$ be the unique inverse of $s$ in $T$. Clearly $s^{\circ} \in A(s) \cap T$. Now let $t \in A(s) \cap T$. Then $t s t \in V(s) \cap T$ and so $s^{\circ}=t s t$, giving $s^{\circ} \leq t$.

Conversely, let $T$ be an associate inverse subsemigroup of $S$ satisfying $t S t \subseteq T$, for all $t \in T$. Let $s \in S$ and $s^{*}$ be the least associate of $s$ in $T$. Since $s^{*} s s^{*} \in V(s) \cap T$ and $s^{*} s s^{*} \leq s^{*}$, we obtain $s^{*}=s^{*} s s^{*}$, whence $s^{*} \in V(s)$. Now let $t \in V(s) \cap T$. From $s^{*} \leq t$ we obtain $s^{*}=e t=t f$, for some idempotents $e, f \in T$. We have

$$
t s s^{*}=t s t f=t f=s^{*}
$$

and

$$
s^{*} s t=e t s t=e t=s^{*} .
$$

So,

$$
s^{*}=t s s^{*}=t s s^{*} s t=t s t=t .
$$


Thus $s^{*}$ is the unique inverse of $s$ in $T$.

In view of this proposition, it follows from [[1], Corollary 1] that in a regular semigroup any two associate inverse subsemigroups of $S$ satisfying (1) are isomorphic. By contrast with inverse transversals, this cannot be generalized to any two associate inverse subsemigroups of $S$ - see Examples 3.1, 3.2 and 3.3, 3.4. Moreover, Example 3.2 shows that an associate inverse transversal does not necessarily satisfy condition (1) of Proposition 3.3.

The next proposition is a consequence of Theorem 2.1 and [[2], Theorem 1.12].

Proposition 3.4. Let $S$ be a regular semigroup with a unary operation $x \mapsto x^{\prime}$ satisfying

(1) $s s^{\prime} s=s$;

(2) $s^{\prime} s^{\prime \prime} t^{\prime} t^{\prime \prime}=t^{\prime} t^{\prime \prime} s^{\prime} s^{\prime \prime}$

(3) $(s t)^{\prime}=t^{\prime} s^{\prime}$;

(4) $s^{\prime \prime \prime}=s^{\prime}$.

If $S^{\prime} \cap V(x) \neq \emptyset$, then $S^{\prime}$ is an inverse transversal of $S$ and $S$ is orthodox.

Conversely, if $S$ is an orthodox semigroup with an inverse transversal $S^{\circ}$, say, then the assignment $x \mapsto x^{\circ}$ is a unary operation that satisfies conditions (1), (2), (3) and (4).

Proof. Suppose first that $S$ is a regular semigroup with a unary operation $x \mapsto x^{\prime}$ satisfying the given conditions. Let $S^{\prime}=\left\{x^{\prime}: x \in S\right\}$. By (3), $S^{\prime}$ is a semigroup and, by (1), for each $x \in S, x^{\prime} \in S^{\prime} \cap A(x)$. We show that $x^{\prime}$ is the least element of $A(x) \cap S^{\prime}$. We have

$$
\begin{array}{rlrl}
t^{\prime} \in S^{\prime} \cap A(x) & \Rightarrow x=x t^{\prime} x & \\
& \Rightarrow x^{\prime}=x^{\prime} t^{\prime \prime} x^{\prime} & & \text { by }(3) \\
& \Rightarrow x^{\prime}=x^{\prime} t^{\prime \prime} t^{\prime \prime \prime} t^{\prime \prime} x^{\prime} & & \text { by }(1) \\
& \Rightarrow x^{\prime}=x^{\prime} t^{\prime \prime} t^{\prime} t^{\prime \prime} x^{\prime} & & \text { by }(4) \\
& \Rightarrow x^{\prime} x^{\prime \prime}=x^{\prime} t^{\prime \prime} t^{\prime \prime} t^{\prime} x^{\prime \prime} & \\
& \Rightarrow x^{\prime} x^{\prime \prime}=x^{\prime} t^{\prime \prime} x^{\prime} x^{\prime \prime} t^{\prime} t^{\prime \prime} & \text { by }(1),(2) \\
& \Rightarrow x^{\prime} x^{\prime \prime} x^{\prime}=x^{\prime} t^{\prime \prime} x^{\prime} x^{\prime \prime} t^{\prime \prime} t^{\prime} & \\
& \Rightarrow x^{\prime}=x^{\prime} x^{\prime \prime} t^{\prime} t^{\prime \prime} x^{\prime} & \\
& \Rightarrow x^{\prime}=t^{\prime} t^{\prime \prime} x^{\prime} x^{\prime \prime} x^{\prime} . & & \text { by }(2) \\
& \Rightarrow x^{\prime}=t^{\prime} t^{\prime \prime} x^{\prime} . &
\end{array}
$$

Dually, we have $x^{\prime}=x^{\prime} t^{\prime \prime} t^{\prime}$. Since $t^{\prime \prime} x^{\prime}$ and $x^{\prime} t^{\prime \prime}$ are idempotents, $x^{\prime} \leq t^{\prime}$. By Theorem 2.1, it follows that the semigroup $S^{\prime}$ is inverse.

Let $x \in S$ and suppose that $V(x) \cap S^{\prime} \neq \emptyset$. We show that $\left|V(x) \cap S^{\prime}\right|=1$. Let $t^{\prime} \in V(x)$. Since $t^{\prime} \in A(x)$ we have $x^{\prime} \leq t^{\prime}$ and so $x^{\prime}=e t^{\prime}=t^{\prime} f$, for some idempotents $e$ and $f$ in $S$. 
Also,

$$
t^{\prime} x x^{\prime}=t^{\prime} x t^{\prime} f=t^{\prime} f=x^{\prime}
$$

and

$$
x^{\prime} x t^{\prime}=e t^{\prime} x t^{\prime}=e t^{\prime}=x^{\prime}
$$

Hence,

$$
x^{\prime}=t^{\prime} x x^{\prime}=t^{\prime} x x^{\prime} x t^{\prime}=t^{\prime} x t^{\prime}=t^{\prime} .
$$

Finally, by [[2], Theorem 1.12], $S$ is orthodox.

Conversely, suppose that $S$ is an orthodox semigroup with inverse transversal $S^{\circ}=$ $\left\{x^{\circ}: x \in S\right\}$, where $\left\{x^{\circ}\right\}=V(x) \cap S^{\circ}$ for each $x \in S$. Consider the unary operation $x \mapsto x^{\circ}$ defined in $S$. Let $s, t \in S$. Clearly $s=s s^{\circ} s$. Also, since $S^{\circ}$ is inverse, $x^{\circ \circ \circ}=$ $\left(\left(x^{\circ}\right)^{-1}\right)^{-1}=x^{\circ}$ and the idempotents of $S^{\circ}$ commute, whence (4) and (2) hold. Condition (3) is an immediate consequence of [[2], Theorem 1.12].

Unlikely to the class of regular non-orthodox semigroups, inverse transversals and associate inverse semigroups are related for orthodox semigroups. In fact, as an immediate consequence of Proposition 3.4 and its proof, we have

Proposition 3.5. Every inverse transversal of an orthodox semigroup is associate.

Example 3.1 below shows that the converse of Proposition 3.5 does not hold.

Example 3.1. Let $S=\{1, a, b, c, d\}$ be the band with operation defined by

\begin{tabular}{l|lllll} 
& 1 & $a$ & $b$ & $c$ & $d$ \\
\hline 1 & 1 & $a$ & $b$ & $c$ & $d$ \\
$a$ & $a$ & $a$ & $b$ & $a$ & $a$ \\
$b$ & $b$ & $a$ & $b$ & $b$ & $b$ \\
$c$ & $c$ & $a$ & $b$ & $c$ & $d$ \\
$d$ & $d$ & $a$ & $b$ & $c$ & $d$
\end{tabular}.

Let $x^{*}=1$, for all $x \in S$. Then $S^{*}$ is an associate inverse subsemigroup and is not an inverse transversal of $S$.

Example 3.2. Let $S$ be the band defined in Example 3.1. Define a unary operation $*$ in $S$ by

$$
1^{*}=1, \quad a^{*}=b^{*}=b, \quad c^{*}=d^{*}=c .
$$

Then $S^{*}=\{1, b, c\}$ is an associate inverse transversal of $S$. Moreover, since cac $=a \notin S^{*}$, $S^{*}$ does not satisfy condition (1) of Proposition 3.3.

Example 3.3. Let $A_{2}=\left\langle a, b \mid a^{2}=a b a=a, b a b=b, b^{2}=0\right\rangle=\{a, b, a b, b a, 0\}$ be the 5element non-orthodox 0-simple semigroup. Let $A_{2}^{*}=\{a\}$. Since, for all $x \in A_{2}, a \in A(x)$ and $\left|A(x) \cap A_{2}^{*}\right|=1, A_{2}^{*}$ is an associate inverse subsemigroup of $A_{2}$. Since $a \notin V(0), A_{2}^{*}$ is not an inverse transversal of $A_{2}$. 
Example 3.4. Let $A_{2}$ be the non-orthodox semigroup referred to in Example 3.3. Let $*$ be the unary operation defined in $A_{2}$ by

$$
a^{*}=(b a)^{*}=(a b)^{*}=b^{*}=a, \quad 0^{*}=0 .
$$

Then $A_{2}^{*}=\{a, 0\}$ is an associate inverse transversal of $A_{2}$.

Examples 3.1, 3.2 and 3.3, 3.4 show that two associate inverse subsemigroups of a semigroup are not necessarily isomorphic.

Example 3.5. Let $C^{1}$ be a non-orthodox completely simple semigroup with identity 1 adjoined. Since $C$ is non-orthodox, there exist maximal subgroups $G$ and $H$ such that the unique inverse of the identity element e of $G$ in $H$ is not equal to the identity of $H$, i.e., it is not an idempotent in $H$. Let $\left(C^{1}\right)^{o}=H \cup\{1\}$. Then $\left(C^{1}\right)^{o}$ is an inverse transversal of $C^{1}$ such that, for the idempotent $e \in G, e^{o}$ is not an idempotent. So, by Corollary 3.2, the inverse transversal $\left(C^{1}\right)^{o}$ is not associate.

Example 3.6. [[2], Example 3.3] Let $S$ be the set of real singular $2 \times 2$ matrices having a non-zero element in $(1,1)$-position. Let $M$ consist of $S$ with the $2 \times 2$ zero matrix adjoined. Then $M$ is a regular semigroup and, relative to the definitions

$$
\left[\begin{array}{cc}
a & b \\
c & a^{-1} b c
\end{array}\right]^{\circ}=\left[\begin{array}{cc}
a^{-1} & 0 \\
0 & 0
\end{array}\right],\left[\begin{array}{ll}
0 & 0 \\
0 & 0
\end{array}\right]^{\circ}=\left[\begin{array}{ll}
0 & 0 \\
0 & 0
\end{array}\right] \text { and } I_{2}^{\circ}=I_{2},
$$

the set $\left(M^{1}\right)^{\circ}=\left\{\left[\begin{array}{ll}x & 0 \\ 0 & 0\end{array}\right]: x \neq 0\right\} \cup\left\{\left[\begin{array}{ll}0 & 0 \\ 0 & 0\end{array}\right]\right\} \cup\left\{I_{2}\right\}$ is an inverse transversal of $M^{1}$. Let $X$ be the $2 \times 2$ matrix having $\frac{1}{2}$ in each position. Then $X \in E\left(M^{1}\right)$ and $X^{\circ}=\left[\begin{array}{ll}2 & 0 \\ 0 & 0\end{array}\right]$. Obviously $X^{\circ} \notin E\left(M^{1}\right)$. Again, by Corollary 3.2, we conclude that the inverse transversal $\left(M^{1}\right)^{\circ}$ is not associate.

The next example shows that there are associate inverse subsemigroups of a semigroup for which condition (3) of Proposition 3.4 is not satisfied.

Example 3.7. Let $B$ be the following semilattice:

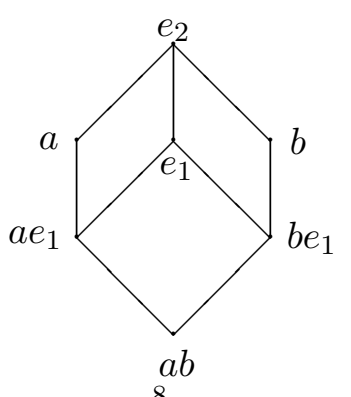


Then $B$ has the two element chain $\left\{e_{1}, e_{2}\right\}$, with $e_{1}<e_{2}$, as associate subsemilattice. Since $a e_{1} a \neq a$ and $b e_{1} b \neq b$, we get $a^{*}=e_{2}=b^{*}$. However

$$
a b e_{1} a b=a b \Rightarrow(a b)^{*}=e_{1}<e_{2}=b^{*} a^{*} .
$$

\section{The structure theorem}

In this section we proceed with the description of a method of constructing regular semigroups with an associate inverse subsemigroup. As seen in Proposition 2.3, such a semigroup $S$ satisfies condition $x^{*} y^{*}=\left(x^{*} y^{*}\right)^{* *}$, for all $x, y \in S$. However, condition $(x y)^{*}=y^{*} x^{*}$ is not necessarily satisfied for all $x, y \in S$ (see Example 3.7). Moreover, the latter condition together with $e f^{*} e^{*}=e f^{*}$ and $e^{*} f^{*} e=f^{*} e, \forall e, f \in E(C)$ allows $S^{*}$ to act on $C^{*} C C^{*}$, where $C$ denotes the subsemigroup generated by the idempotents of $S$, by conjugation. This proved to be crucial for our construction and so our goal in this section is to describe all regular semigroups $S$, with an associate inverse subsemigroup, that satisfy

(I1) $(s t)^{*}=t^{*} s^{*}, \forall s, t \in S$;

(I2) $e f^{*} e^{*}=e f^{*}$ and $e^{*} f^{*} e=f^{*} e, \forall e, f \in E(S)$.

The construction we present is somehow semidirect product like because of the multiplication formula, where products are formed of an element and the result of the action on another element. Obtaining Theorems 3 and 4, due to Blyth and Mendes Martins [3], as an application of our construction, was a natural consequence.

Let $C$ be an idempotent generated semigroup with associate inverse subsemigroup $C^{*}$ satisfying (I1) and (I2).

Notice that it follows from (I1) that $C^{*}$ is a semilattice since for $e \in E(C)$ we get $e^{*}=\left(e^{2}\right)^{*}=e^{*} e^{*}$ whence, for arbitrary $c=e_{1} \ldots e_{n} \in C, e_{i} \in E(C)$, we have, by (I1), $c^{*}=e_{n}^{*} \ldots e_{1}^{*} \in E(C)$. In particular, each $c^{*}$ is equal to $c^{* *}$. Moreover, for every $c, d \in C$ we have

$$
c d^{*} c^{*}=c c^{*} c d^{*}\left(c^{*} c\right)^{*}=c c^{*} c d^{*}=c d^{*}
$$

and dually $c^{*} d^{*} c=d^{*} c$. Further, each $c d^{*} \in C C^{*}$ equals $c d^{*}\left(c d^{*}\right)^{*}$ since $c d^{*}\left(c d^{*}\right)^{*}=$ $c d^{*} d^{* *} c^{*}=c d^{*}$. Similarly, we have $d^{*} c=\left(d^{*} c\right)^{*} d^{*} c$. Then every element $y$ of $C C^{*}$ (respectively, $C^{*} C$ ) is such that $y=y y^{*}$ (respectively, $y=y^{*} y$ ). We can now prove the following lemma:

Lemma 4.1. Every element $x \in C^{*} C C^{*}$ can be written as $x=c^{*} c c^{*}$ for some $c \in C$.

Proof. Let $x=d^{*} c e^{*} \in C^{*} C C^{*}$. Then $x \in C \cap C C^{*} \cap C^{*} C$ and so,

$$
x^{*} x x^{*}=x^{*}\left(x x^{*}\right)=x^{*} x=x .
$$

The result follows. 
Lemma 4.2. Let $S$ be a regular semigroup with associate inverse subsemigroup $S^{*}$, satisfying $(s t)^{*}=t^{*} s^{*}$ for all $s, t \in S$. Then condition (I2) is equivalent to

(I2)' $a^{*} b c^{*} d^{*}=d^{*} a^{*} b c^{*}$ for all $a, b, c, d \in\langle E(S)\rangle$.

Proof. Note first that $\langle E(S)\rangle \cap S^{*}=E\left(S^{*}\right)$.

$(\mathrm{I} 2)^{\prime} \Rightarrow(\mathrm{I} 2)$. For $e, f \in E(S)$ we have

$$
e f^{*}=e e^{*} e^{*} e f^{*}=e e^{*} e f^{*} e^{*}=e f^{*} e^{*}
$$

and dually $f^{*} e=e^{*} f^{*} e$.

(I2) $\Rightarrow$ (I2)'. Let first $e \in E(S), a, c, d \in\langle E(S)\rangle$. Then

$$
\begin{aligned}
d^{*} a^{*} e c^{*} & =d^{*} a^{*} e c^{*}\left(d^{*} a^{*} e\right)^{*}=d^{*} a^{*} e c^{*}\left(a^{*} e\right)^{*} d^{*}=d^{*} a^{*} e c^{*} d^{*} \\
& =d^{*}\left(e c^{*}\right)^{*} a^{*} e c^{*} d^{*}=\left(e c^{*} d^{*}\right)^{*} a^{*} e c^{*} d^{*}=a^{*} e c^{*} d^{*} .
\end{aligned}
$$

Consider now $b \in\langle E(S)\rangle$. Note that $b^{*}, b b^{*}, b^{*} b \in E(S)$. We get

$$
a^{*} b c^{*} d^{*}=a^{*} b b^{*} b^{*} b^{*}\left(b^{*} b\right) c^{*} d^{*}=a^{*} b b^{*} b^{*} d^{*} b^{*} b^{*} b c^{*}=d^{*} a^{*} b b^{*} b^{*} b c^{*}=d^{*} a^{*} b c^{*},
$$

which completes our proof.

Let now $V$ be an inverse semigroup acting on $C^{*} C C^{*}$ by endomorphisms on the left such that:

(A1) the action is compatible with the unary operation $*$, i.e., $\left({ }^{g}\left(c^{*} c c^{*}\right)\right)^{*}={ }^{g}\left(c^{*}\right)$, for all $g \in V, c \in C$;

(A2) there is a surjective homomorphism $\pi: C^{*} C C^{*} \rightarrow E(V)$ such that, for all $e \in E(V)$ and $c \in C$,

$$
{ }^{e}\left(c^{*} c c^{*}\right)=c^{*} c c^{*} \Longleftrightarrow\left(c^{*} c c^{*}\right) \pi \leq e
$$

and $\pi$ is injective on $C^{*}$. In particular, this means that $C^{*} \simeq E(V)$.

Lemma 4.3. For all $c \in C,\left(c^{*} c c^{*}\right) \pi=c^{*} \pi$.

Proof. Let $c \in C$. Since ${ }^{\left(c^{*} c c^{*}\right) \pi}\left(c^{*} c c^{*}\right)=c^{*} c c^{*}$, we have

$$
c^{*}=\left(c^{*} c c^{*}\right)^{*}=\left(\left(c^{*} c c^{*}\right) \pi\left(c^{*} c c^{*}\right)\right)^{*}=\left(c^{*} c c^{*}\right) \pi\left(c^{*}\right)
$$

which yields

$$
c^{*} \pi \leq\left(c^{*} c c^{*}\right) \pi=c^{*} \pi\left(c^{*} c c^{*}\right) \pi \leq c^{*} \pi,
$$

whence $c^{*} \pi=\left(c^{*} c c^{*}\right) \pi$.

Lemma 4.4. Let $g \in V, n>1$ and $c_{1}, c_{2}, \ldots, c_{n} \in C^{*} C C^{*}$ such that ${ }^{g g^{-1}} c_{i}=c_{i}$ for some $i \in\{1,2, \ldots, n\}$. Then

$$
g^{-1}\left(c_{1} c_{2} \cdots c_{n}\right)=c_{1} c_{2} \cdots c_{n} .
$$


Proof. If $g^{-1}\left(c_{i}\right)=c_{i}$ we have $c_{i} \pi \leq g g^{-1}$ and so

$$
g g^{-1} c_{i} \pi=c_{i} \pi
$$

Thus

$$
\begin{aligned}
& g g^{-1}\left(c_{1} c_{2} \cdots c_{i} \cdots c_{n}\right)=g g^{-1}\left(\left(c_{1} \cdots c_{i} \cdots c_{n}\right) \pi\left(c_{1} \cdots c_{i} \cdots c_{n}\right)\right) \\
&=g g^{-1} c_{1} \pi \cdots c_{i} \pi \cdots c_{n} \pi \\
&\left.=c_{1} \cdots c_{i} \cdots c_{n}\right) \\
&={ }^{c_{1} \pi \cdots g g^{-1} c_{i} \pi \cdots c_{n} \pi}\left(c_{1} \cdots c_{i} \cdots c_{n}\right)={ }^{c_{1} \pi \cdots c_{i} \pi \cdots c_{n} \pi}\left(c_{1} \cdots c_{i} \cdots c_{n}\right) \\
&\left(c_{1} \cdots c_{i} \cdots c_{n}\right) \pi \\
&
\end{aligned}
$$

Lemma 4.5. Let $g \in V$ and $c \in C^{*} C C^{*}$ such that ${ }^{g g^{-1}} c=c$. Then,

$$
g^{-1}(c \pi) g=\left(g^{-1} c\right) \pi \text {. }
$$

Proof. First, we notice that, for every $a \in E(V)$, we have

$$
{ }^{a}\left(g^{-1} c\right)=g^{-1} c \Longrightarrow g^{-1}(c \pi) g \leq a
$$

In fact,

$$
\begin{aligned}
& { }^{a}\left(g^{-1} c\right)=g^{-1} c \Longrightarrow g^{a g^{-1}} c=g g^{-1} c \\
& \Longrightarrow{ }^{g a g^{-1}} c=c \\
& \Longrightarrow c \pi \leq g a g^{-1} \quad \text { by definition of } \pi \\
& \Longrightarrow g^{-1}(c \pi) g \leq g^{-1} g a g^{-1} g \\
& \Longrightarrow g^{-1}(c \pi) g \leq a \text {. }
\end{aligned}
$$

Since $\left(g^{-1} c\right) \pi \in E(V)$ and, by definition of $\pi,\left(g^{-1} c\right) \pi\left(g^{-1} c\right)=g^{-1} c$, we have, by (2), that

$$
g^{-1}(c \pi) g \leq\left(g^{-1} c\right) \pi
$$

Moreover, again by hypothesis and by definition of $\pi$, we have

$$
g^{-1}(c \pi) g\left(g^{-1} c\right)=g^{-1}(c \pi)\left(g^{-1} c\right)=g^{-1}\left({ }^{(c \pi)} c\right)=g^{-1} c .
$$

Thus, since $g^{-1}(c \pi) g \in E(V)$, we have

$$
\left(g^{-1} c\right) \pi \leq g^{-1}(c \pi) g
$$

By (3) and (4) equality follows.

Using the action and the homomorphism defined in (A1) and (A2), we now consider the following construction: Let

$$
[C, V]=\left\{\left(a a^{*}, g, b^{*} b\right): a, b \in C, g \in V, a^{*} \pi=g g^{-1}, b^{*} \pi=g^{-1} g,{ }^{g}\left(b^{*} b b^{*}\right)=a^{*} a a^{*}\right\} .
$$

Note that if $a^{*} \pi=g g^{-1}$ and $b^{*} \pi=g^{-1} g$,

$$
{ }^{g}\left(b^{*} b b^{*}\right)=a^{*} a a^{*} \Longleftrightarrow{ }^{-1}\left(a^{*} a a^{*}\right)=b^{*} b b^{*} .
$$


In fact,

$$
{ }^{g}\left(b^{*} b b^{*}\right)=a^{*} a a^{*} \Rightarrow g^{-1} g\left(b^{*} b b^{*}\right)=g^{-1}\left(a^{*} a a^{*}\right) \Leftrightarrow b^{*} b b^{*}=g^{-1}\left(a^{*} a a^{*}\right) .
$$

By symmetry, we have equivalence.

Define a multiplication in $[C, V]$ by

$$
\left(a a^{*}, g, b^{*} b\right)\left(c c^{*}, h, d^{*} d\right)=\left(a a^{* g}\left(b^{*} b c c^{*}\right), g h, h^{-1}\left(b^{*} b c c^{*}\right) d^{*} d\right) .
$$

Proposition 4.6. $[C, V]$ is a regular semigroup with

$$
E([C, V])=\left\{\left(a a^{*}, g g^{-1}, b^{*} b\right) \in[C, V]: a a^{*} \in V\left(b^{*} b\right)\right\} .
$$

Proof. We first show that the multiplication is uniquely defined. Let $u=a a^{* g}\left(b^{*} b c c^{*}\right)$. Then $u^{*}=a^{* g}\left(b^{*} c^{*}\right)$ by (i) and (a) and $u=u u^{*}$ by (ii). In order to show that $u^{*} \pi=$ $g h h^{-1} g^{-1}$ it suffices to verify that $\left({ }^{g}\left(b^{*} c^{*}\right)\right) \pi=g h h^{-1} g^{-1}$, since $a^{*} \pi=g g^{-1}$. Since $b^{*} \pi=g^{-1} g$, by Lemma 4.4, we have ${ }^{g^{-1} g}\left(b^{*} c^{*}\right)=b^{*} c^{*}$ and so, by Lemma $4.5,\left({ }^{g}\left(b^{*} c^{*}\right)\right) \pi=$ $g\left(b^{*} c^{*}\right) \pi g^{-1}$. Hence,

$$
\left({ }^{g}\left(b^{*} c^{*}\right)\right) \pi=g g^{-1} g h h^{-1} g^{-1}=g h h^{-1} g^{-1} .
$$

Let $v=h^{-1}\left(b^{*} b c c^{*}\right) d^{*} d$. By a similar argument we obtain $v^{*} \pi=h^{-1} g^{-1} g h$.

Next we observe that $u^{*} u u^{*}=a^{*} a a^{* g}\left(b^{*} b c c^{*}\right)$ and $v^{*} v v^{*}=h^{-1}\left(b^{*} b c c^{*}\right) d^{*} d d^{*}$ by the remarks at the beginning of this section. We compute

$$
\begin{array}{rlr}
g h\left(v^{*} v v^{*}\right) & =g h\left(h^{-1}\left(b^{*} b c c^{*}\right) d^{*} d d^{*}\right)=g h h^{-1}\left(b^{*} b c c^{*}\right)^{g h}\left(d^{*} d d^{*}\right) & \\
& =g h h^{-1}\left(b^{*} b c c^{*}\right) g h h^{-1}\left(c^{*} c c^{*}\right)=g h h^{-1}\left(b^{*} b c c^{*}\right) & \\
& ={ }^{g}\left(h h^{-1}\left(b^{*} b c c^{*}\right)\right)={ }^{g}\left(b^{*} b c c^{*}\right) & \text { by Lemma } 4.3 \\
& ={ }^{g}\left(b^{*} b b^{*}\right)^{g}\left(b^{*} b c c^{*}\right)=a^{*} a a^{* g}\left(b^{*} b c c^{*}\right)=u^{*} u u^{*} . &
\end{array}
$$

We continue showing that the multiplication is associative. Consider the products $\left(\left(a a^{*}, g, b^{*} b\right)\left(c c^{*}, h, d^{*} d\right)\right)\left(e e^{*}, k, f^{*} f\right)$ and $\left(a a^{*}, g, b^{*} b\right)\left(\left(c c^{*}, h, d^{*} d\right)\left(e e^{*}, k, f^{*} f\right)\right)$. The first components of the products are

$$
\left(a a^{* g}\left(b^{*} b c c^{*}\right)^{g h}\left(h^{-1}\left(b^{*} b c c^{*}\right) d^{*} d e e^{*}\right) \text { and } a a^{* g}\left(b^{*} b c c^{* h}\left(d^{*} d e e^{*}\right)\right),\right.
$$

which are in fact equal, since $h h^{-1}\left(b^{*} b c c^{*}\right)=b^{*} b c c^{*}$ by Lemma 4.4. By a similar argument one shows that also the third components coincide. Here the equality ${ }^{h^{-1} h}\left(d^{*} d e e^{*}\right)=$ $d^{*} d e e^{*}$ is used, which comes from ${ }^{h^{-1}}\left(c^{*} c c^{*}\right)=d^{*} d d^{*}$. The equality of the second components is trivial.

Let $\left(a a^{*}, g, b^{*} b\right) \in[C, V]$. Then, from ${ }^{g}\left(b^{*} b b^{*}\right)=a^{*} a a^{*}$ and ${ }^{g^{-1}}\left(a^{*} a a^{*}\right)=b^{*} b b^{*}$ we have $a^{*}=\left({ }^{g}\left(b^{*} b b^{*}\right)\right)^{*}={ }^{g}\left(b^{*}\right)$ and $b^{*}=\left(g^{-1}\left(a^{*} a a^{*}\right)\right)^{*}=g^{-1}\left(a^{*}\right)$. Thus, since $a^{*} \pi=g g^{-1}$ and 
$b^{*} \pi=g^{-1} g$, the element $\left(b^{*}, g^{-1}, a^{*}\right)$ is in $[C, V]$. Moreover,

$$
\begin{aligned}
\left(a a^{*}, g, b^{*} b\right)\left(b^{*}, g^{-1}, a^{*}\right)\left(a a^{*}, g, b^{*} b\right) & =\left(a a^{*} g\left(b^{*} b b^{*}\right), g g^{-1}, g\left(b^{*} b b^{*}\right) a^{*}\right)\left(a a^{*}, g, b^{*} b\right) \\
& =\left(a a^{*}, g g^{-1}, a^{*} a a^{*}\right)\left(a a^{*}, g, b^{*} b\right) \\
& =\left(a a^{*} g g^{-1}\left(a^{*} a a^{*}\right), g, g^{-1}\left(a^{*} a a^{*}\right) b^{*} b\right) \\
& =\left(a a^{*} a^{*} a a^{*}, g, b^{*} b b^{*} b^{*} b\right) \\
& =\left(a a^{*}, g, b^{*} b\right) .
\end{aligned}
$$

Hence, $[C, V]$ is regular.

Finally, we prove that every idempotent of $[C, V]$ is of the form $\left(a a^{*}, g g^{-1}, b^{*} b\right)$ with $a a^{*} \in V\left(b^{*} b\right)$. That the second component of an element belonging to $E([C, V])$ must be an idempotent in $V$ is clear. Since, by Lemma $4.4,{ }^{g g^{-1}}\left(b^{*} b a a^{*}\right)=b^{*} b a a^{*}$, we have

$$
\begin{aligned}
\left(a a^{*}, g g^{-1}, b^{*} b\right)\left(a a^{*}, g g^{-1}, b^{*} b\right)=\left(a a^{*}, g g^{-1}, b^{*} b\right) & \Leftrightarrow\left\{\begin{array}{l}
a a^{*} b^{*} b a a^{*}=a a^{*} \\
b^{*} b a a^{*} b^{*} b=b^{*} b
\end{array}\right. \\
& \Leftrightarrow a a^{*} \in V\left(b^{*} b\right) .
\end{aligned}
$$

Proposition 4.7. The set $\left\{\left(a a^{*}, v, b^{*} b\right) \in[C, V]: v \in E(V)\right\}$ is the semigroup generated by the idempotents of $[C, V]$. Moreover, $\langle E([C, V])\rangle \simeq C$.

Proof. On one hand, since $V$ is an inverse semigroup, the second component of the product of a finite number of idempotents of $[C, V]$ is always an idempotent.

On the other hand, for $a, b \in C$ and $g \in V$ such that $\left(a a^{*}, g g^{-1}, b^{*} b\right) \in[C, V]$, the elements $\left(a a^{*}, a^{*} \pi, a^{*} a a^{*}\right)$ and $\left(b^{*} b b^{*}, b^{*} \pi, b^{*} b\right)$ are idempotents of $[C, V]$ and

$$
\left(a a^{*}, g g^{-1}, b^{*} b\right)=\left(a a^{*}, g g^{-1}, a^{*} a a^{*}\right)\left(b^{*} b b^{*}, g g^{-1}, b^{*} b\right) .
$$

So, equality follows.

Next, we show that the mapping $\theta: c \mapsto\left(c c^{*}, c^{*} \pi, c^{*} c\right)$ is an isomorphism from $C$ onto $\langle E([C, V])\rangle$. For any $c, d \in C$,

$$
\begin{aligned}
c \theta d \theta & =\left(c c^{*}, c^{*} \pi, c^{*} c\right)\left(d d^{*}, d^{*} \pi, d^{*} d\right) \\
& =\left(c c^{*} c^{*} \pi\left(c^{*} c d d^{*}\right), c^{*} \pi d^{*} \pi, d^{*} \pi\left(c^{*} c d d^{*}\right) d^{*} d\right) \\
& =\left(c c^{*} c^{*} c d d^{*},\left(d^{*} c^{*}\right) \pi, c^{*} c d d^{*} d^{*} d\right) \\
& =\left(c d(c d)^{*},(c d)^{*} \pi,(c d)^{*} c d\right)=(c d) \theta
\end{aligned}
$$

by Lemma 4.2

and so $\theta$ is a homomorphism. Also, if $c c^{*}=d d^{*}$ and $c^{*} c=d^{*} d$ then

$$
c=c c^{*} c^{*} c=d d^{*} d^{*} d=d .
$$


Finally, given $\left(a a^{*}, g g^{-1}, b^{*} b\right) \in\langle E([C, V])\rangle$, we have

$$
\begin{aligned}
\left(a a^{*}, g g^{-1}, b^{*} b\right) & =\left(a a^{*}, a^{*} \pi, a^{*} a a^{*}\right)\left(b^{*} b b^{*}, b^{*} \pi, b^{*} b\right) \\
& =\left(a a^{*}\left(a a^{*}\right)^{*}, a^{*} \pi,\left(a a^{*}\right)^{*} a a^{*}\right)\left(\left(b^{*} b\right)^{*} b^{*} b\left(b^{*} b\right)^{*}, b^{*} \pi,\left(b^{*} b\right)^{*} b^{*} b\right) \\
& =\left(a a^{*}\right) \theta\left(b^{*} b\right) \theta \\
& =\left(a a^{*} b^{*} b\right) \theta .
\end{aligned}
$$

Thus $\theta$ is a bijection.

Proposition 4.8. The set $\left\{\left(a^{*}, g, b^{*}\right) \mid\left(b b^{*}, g^{-1}, a^{*} a\right) \in[C, V]\right\}$ is an associate inverse subsemigroup of $[C, V]$ which is isomorphic to $V$.

Proof. Let $K=\left\{\left(a^{*}, g, b^{*}\right) \mid\left(b b^{*}, g^{-1}, a^{*} a\right) \in[C, V]\right\}$. Obviously $K$ is a subsemigroup of $[C, V]$. We show that the mapping $\psi: K \rightarrow V$ defined by $\left(a^{*}, g, b^{*}\right) \mapsto g$ is an isomorphism: $\psi$ is trivially uniquely defined and a homomorphism. The mapping $\psi$ is injective since $\left(a^{*}, g, b^{*}\right),\left(c^{*}, g, d^{*}\right) \in[C, V]$ implies $a^{*} \pi=g g^{-1}=c^{*} \pi$ and $b^{*} \pi=g^{-1} g=$ $d^{*} \pi$, whence $a^{*}=c^{*}, b^{*}=d^{*}$ follow by injectivity of $\pi$ on $C^{*}$. It remains to show that $\psi$ is surjective. Let $g \in V$. Since $\pi$ is surjective, there is $c^{*} c c^{*} \in C^{*} C C^{*}$ such that $\left(c^{*} c c^{*}\right) \pi=g^{-1} g$. Consider $\left(c^{*} c c^{*}, g^{-1}, c^{*} c c^{*}\right)$. Since ${ }^{-1} g\left(c^{*} c c^{*}\right)=c^{*} c c^{*}$, it follows by Lemma 4.5 that $\left({ }^{g}\left(c^{*} c c^{*}\right)\right) \pi=g\left(c^{*} c c^{*}\right) \pi g^{-1}$. We conclude that $\left({ }^{g}\left(c^{*} c c^{*}\right)\right) \pi=g g^{-1}$ and then $\left(c^{*} c c^{*}, g^{-1},{ }^{g}\left(c^{*} c c^{*}\right)\right) \in[C, V]$. Therefore,

$$
\left(\left({ }^{g}\left(c^{*} c c^{*}\right)\right)^{*}, g,\left(c^{*} c c^{*}\right)^{*}\right)=\left(c^{*} c c^{*}, g^{-1},{ }^{g}\left(c^{*} c c^{*}\right)\right)^{*} \in K .
$$

Hence $\psi$ is surjective and it is clear that each element $\left(a a^{*}, g, b^{*} b\right)$ of $[C, V]$ has a least associate in $K$, namely $\left(b^{*}, g^{-1}, a^{*}\right)$.

As a consequence of the proof of the previous proposition, for each $\left(a a^{*}, g, b^{*} b\right) \in[C, V]$, $\left(a a^{*}, g, b^{*} b\right)^{*}=\left(b^{*}, g^{-1}, a^{*}\right)$ and so, $\left\{\left(a^{*}, g, b^{*}\right) \mid\left(b b^{*}, g^{-1}, a^{*} a\right) \in[C, V]\right\}=[C, V]^{*}$.

Note that the idempotents of $[C, V]^{*}$ are of the form $\left(a^{*}, g g^{-1}, a^{*}\right)$. Further, if we drop the assumption that $\pi$ is injective on $C^{*}$ or surjective on $C^{*} C C^{*}$ then $[C, V]$ is still a semigroup with associate inverse subsemigroup $[C, V]^{*}$. However, $[C, V]^{*}$ is then not necessarily isomorphic to $V$.

Proposition 4.9. The semigroup $[C, V]$ satisfies conditions (I1) and (I2).

Proof. (I1) It follows trivially using the fact that $\left(a a^{*}, g, b^{*} b\right)^{*}=\left(b^{*}, g^{-1}, a^{*}\right)$. 
(I2) Let $\left(a a^{*}, g g^{-1}, b^{*} b\right) \in E([C, V])$ and $\left(c^{*}, h h^{-1}, c^{*}\right) \in E\left([C, V]^{*}\right)$. Then $\left(a a^{*}, g g^{-1}, b^{*} b\right)^{*}=\left(a^{*}, g g^{-1}, a^{*}\right)$. Using Lemma 4.4, we compute

$$
\begin{aligned}
\left(a a^{*}, g g^{-1}, b^{*} b\right)\left(c^{*}, h h^{-1}, c^{*}\right)\left(a^{*}, g g^{-1}, a^{*}\right) & =\left(a a^{*}, g g^{-1}, b^{*} b\right)\left(a^{*} c^{*}, h h^{-1} g g^{-1}, c^{*} a^{*}\right) \\
& =\left(a a^{*} b^{*} b a^{*} c^{*}, g g^{-1} h h^{-1}, b^{*} b a^{*} c^{*}\right) \\
& =\left(a a^{*} b^{*} b c^{*}, g g^{-1} h h^{-1}, b^{*} b c^{*}\right) \text { since } a^{*}=b^{*}, \\
\quad \text { which comes from } a a^{*} \in V\left(b^{*} b\right) & \\
& =\left(a a^{*}, g g^{-1}, b^{*} b\right)\left(c^{*}, h h^{-1}, c^{*}\right) .
\end{aligned}
$$

The rest of the proof follows by duality.

We can now state the main result of this paper.

Theorem 4.10. Let $C$ be an idempotent generated semigroup and $V$ an inverse semigroup satisfying conditions (I1) and (I2). Then the set

$$
[C, V]=\left\{\left(a a^{*}, g, b^{*} b\right): a, b \in C, g \in V, a^{*} \pi=g g^{-1}, b^{*} \pi=g^{-1} g,{ }^{g}\left(b^{*} b b^{*}\right)=a^{*} a a^{*}\right\},
$$

where the morphism $\pi$ and the action are the ones defined in (A1) and (A2), with multiplication defined by

$$
\left(a a^{*}, g, b^{*} b\right)\left(c c^{*}, h, d^{*} d\right)=\left(a a^{* g}\left(b^{*} b c c^{*}\right), g h, h^{-1}\left(b^{*} b c c^{*}\right) d^{*} d\right),
$$

is a regular semigroup with an associate inverse subsemigroup

$$
[C, V]^{*}=\left\{\left(a^{*}, g, b^{*}\right) \in[C, V]\right\}
$$

that satisfies conditions (I1) and (I2).

Moreover, $[C, V]^{*} \simeq V$ and $\langle E([C, V])\rangle \simeq C$.

Conversely, every regular semigroup $S$ with associate inverse subsemigroup $S^{*}$ satisfying (I1) and (I2) is isomorphic to a semigroup obtained by the preceding construction, where $C=\langle E(S)\rangle$ and $V=S^{*}$.

Proof. The first part is an immediate consequence of Propositions 4.6, 4.7, 4.8 and 4.9.

The semigroup $C$ is of the form as prescribed in the preceding construction. Further, $S^{*}$ acts on $C^{*} C C^{*}$ on the left by conjugation, since

$$
s^{* *} c^{*} c c^{*} s^{*} s^{* *} d^{*} d d^{*} s^{*}=s^{* *} c^{*} c c^{*} d^{*} d d^{*} s^{*}
$$

and

$$
t^{* *} s^{* *} c^{*} c c^{*} s^{*} t^{*}=(t s)^{* *} c^{*} c c^{*}(t s)^{*} .
$$

Moreover this action is compatible with $*$, since

$$
\left(s^{* *}\left(c^{*} c c^{*}\right)\right)^{*}=\left(s^{* *} c^{*} c c^{*} s^{*}\right)^{*}=s_{15}^{* *} c^{*} s^{*}={ }^{* *}\left(c^{*}\right)={ }^{s^{* *}}\left(\left(c^{*} c c^{*}\right)^{*}\right) .
$$


Now define $\pi: C^{*} C C^{*} \rightarrow E\left(S^{*}\right)$ by $\pi: c^{*} c c^{*} \mapsto c^{*}$. Then

$$
\begin{aligned}
s^{* *} s^{*}\left(c^{*} c c^{*}\right)=c^{*} c c^{*} & \Longleftrightarrow s^{* *} s^{*} c^{*} c c^{*}=c^{*} c c^{*} \Longleftrightarrow s^{* *} s^{*} c^{*}=c^{*} \\
& \Longleftrightarrow c^{*} \leq s^{* *} s^{*} \Longleftrightarrow\left(c^{*} c c^{*}\right) \pi \leq s^{* *} s^{*},
\end{aligned}
$$

and, since $C$ satisfies (I1) and (I2), $\pi$ is a homomorphism, which is clearly surjective and injective on $C^{*}$. Hence we may form $[C, V]$.

Finally we show that the mapping $\phi: S \rightarrow[C, V]$, defined by $s \mapsto\left(s s^{*}, s^{* *}, s^{*} s\right)$ is an isomorphism. Since $\left(s s^{*}\right)^{*}=s^{* *} s^{*},\left(s^{*} s\right)^{*}=s^{*} s^{* *}$ and

$$
s^{* *}\left(\left(s^{*} s\right)^{*} s^{*} s\left(s^{*} s\right)^{*}\right)={ }^{* *}\left(s^{*} s^{* *} s^{*} s s^{*} s^{* *}\right)=s^{* *} s^{*} s s^{*} s^{* *} s^{*}=\left(s s^{*}\right)^{*} s s^{*}\left(s s^{*}\right)^{*},
$$

$\phi$ is well defined.

We continue showing that $\phi$ is a homomorphism. Let $s, t \in S$. It follows that

$$
\begin{aligned}
s \phi t \phi & =\left(s s^{*}, s^{* *}, s^{*} s\right)\left(t t^{*}, t^{* *}, t^{*} t\right) \\
& =\left(s s^{*} s^{* *}\left(s^{*} s t t^{*}\right), s^{* *} t^{* *}, t^{*}\left(s^{*} s t t^{*}\right) t^{*} t\right) \\
& =\left(s s^{*} s^{* *} s^{*} s t t^{*} s^{*}, s^{* *} t^{* *}, t^{*} s^{*} s t t^{*} t^{* *} t^{*} t\right) \\
& =\left(s t(s t)^{*},(s t)^{* *},(s t)^{*} s t\right)=(s t) \phi .
\end{aligned}
$$

Moreover, $\phi$ is injective since $s s^{*}=t t^{*}, s^{* *}=t^{* *}$ and $s^{*} s=t^{*} t$ implies

$$
s=s s^{*} s^{* *} s^{*} s=t t^{*} t^{* *} t^{*} t=t .
$$

It remains to show that $\phi$ is surjective. Consider the element $\left(s s^{*}, t^{* *}, u^{*} u\right) \in[C, V]$, i.e., $s^{* *} s^{*}=t^{* *} t^{*}, u^{*} u^{* *}=t^{*} t^{* *}, t^{* *} u^{*} u u^{*} u^{* *} t^{*}=s^{* *} s^{*} s s^{*}$, being equivalent to $t^{* *} u^{*} u t^{*}=$ $s^{* *} s^{*} s s^{*}$ and $t^{*} s^{* *} s^{*} s s^{*} t^{* *}=u^{*} u u^{*} u^{* *}$, being equivalent to $t^{*} s s^{*} t^{* *}=u^{*} u u^{*} u^{* *}$. We obtain

$$
\left(s s^{*} t^{* *} u^{*} u\right) \phi=\left(s s^{*}, t^{* *}, u^{*} u\right)
$$

since

$$
\begin{gathered}
s s^{*} t^{* *} u^{*} u\left(s s^{*} t^{* *} u^{*} u\right)^{*}=s s^{*} t^{* *} u^{*} u u^{*} u^{* *} t^{*} s^{* *} s^{*}=s s^{*} t^{* *} u^{*} u t^{*}=s s^{*} s^{* *} s^{*} s s^{*}=s s^{*}, \\
\left(s s^{*} t^{* *} u^{*} u\right)^{* *}=s^{* *} s^{*} t^{* *} u^{*} u^{* *}=t^{* *} t^{*} t^{* *} t^{*} t^{* *}=t^{* *}
\end{gathered}
$$

and

$$
\left(s s^{*} t^{* *} u^{*} u\right)^{*} s s^{*} t^{* *} u^{*} u=u^{*} u^{* *} t^{*} s^{* *} s^{*} s s^{*} t^{* *} u^{*} u=t^{*} s s^{*} t^{* *} u^{*} u=u^{*} u u^{*} u^{* *} u^{*} u=u^{*} u .
$$

Hence $\phi$ is surjective.

Finally, we observe that, considering the particular case of $S^{*}$ being a quasi-ideal inverse transversal of $S$ (i.e. $S^{*} S S^{*} \subseteq S^{*}$ ), we obtain, as a corollary of Theorem 4.10, the orthodox version of Theorem 4.2 in [7], due to McAlister and McFadden.

Also, Theorem 3 and Theorem 4 in [4] are corollaries of Theorem 4.10. In fact, if $S$ is a regular semigroup with an associate subgroup, then $\langle E(S)\rangle^{*}$ is singleton. Its unique 
element, say $\alpha$, is therefore a medial idempotent of $S$ and so conditions (i) and (ii) in Theorem 4.10 hold (see [4]).

\section{REFERENCES}

[1] T. S. Blyth, J. F. Chen. Inverse transversals are mutually isomorphic. Communications in Algebra 29 (2) (2001), 799-804

[2] T. S. Blyth. Inverse transversals - a guided tour. Proceedings of the International Conference on Semigroups, 18th-23rd June 1999. Braga. World Scientific (Singapore)(2000), 26-43

[3] T. S. Blyth, Emília Giraldes and M. Paula O. Marques-Smith. Associate subgroups of orthodox semigroups. Glasgow Math J. 36 (1994), 163-171

[4] T. S. Blyth, P. Mendes Martins. On associate subgroups of regular semigroups. Communications in Algebra 25 (7) (1997), 2147-2156

[5] J.M. Howie. Fundamentals of semigroup theory. London Math. Soc. Monographs, New Series 12, Oxford University Press, Oxford (1995)

[6] M. Lawson. Inverse semigroups: the theory of partial symmetries. World Scientific, (Singapore) (1998)

[7] D. B. McAlister and R. McFadden. Regular semigroups with inverse transversals. Quart. J. Math. Oxford 34 (1983), 459-474

[8] M. Petrich. Introduction to Semigroups. Charles E. Merrill Publishing Co.; A. Bell and Howell Company. Columbus (1973)

$\begin{array}{llll}\text { Universität Kassel } & \text { U.T.A.D. } & \text { Universidade do Minho } & \text { Universidade do Minho } \\ \text { FB - 17 Mathematik } & \text { Departamento de Matemática } & \text { Centro de Matemática } & \text { Centro de Matemática } \\ \text { D-34127 Kassel, Germany } & \text { 5000 Vila Real, Portugal } & \text { 4710-057 Braga, Portugal } & 4710-057 \text { Braga, Portugal } \\ \text { billhardt@uni-kassel.de } & \text { egs@utad.pt } & \text { psmith@math.uminho.pt } & \text { pmendes@math.uminho.pt }\end{array}$

